\title{
Increasing $3 \alpha, 5 \alpha$-THP following inhibition of neurosteroid biosynthesis in the ventral tegmental area reinstates anti-anxiety, social, and sexual behavior of naturally receptive rats
}

\author{
Cheryl A Frye ${ }^{1,2,3,4}$, Jason J Paris ${ }^{1}$ and Madeline E Rhodes ${ }^{4}$ \\ ${ }^{1}$ Department of Psychology, ${ }^{2}$ Department of Biological Sciences, ${ }^{3}$ The Centers for Neuroscience and ${ }^{4}$ Life Sciences \\ Research 1058, The University at Albany-SUNY, 1400 Washington Avenue, Albany, New York 12222, USA
}

Correspondence should be addressed to C A Frye; Email: cafrye@albany.edu

\begin{abstract}
The progesterone metabolite and neurosteroid, $5 \alpha$-pregnan-3 $\alpha$-ol-20-one ( $3 \alpha, 5 \alpha$-THP), has actions in the midbrain ventral tegmental area (VTA) to modulate lordosis, but its effects on other reproductively relevant behaviors are not well understood. Effects on exploration, anxiety, and social behavior resulting from inhibition of $3 \alpha, 5 \alpha$-THP formation, as well as $3 \alpha, 5 \alpha$-THP enhancement, were investigated in the midbrain VTA. Naturally sexually receptive, female rats $(n=8-10 /$ group) received infusions aimed at the midbrain VTA of vehicle, PK11195 (an inhibitor of neurosteroidogenesis), and/or indomethacin (an inhibitor of $3 \alpha, 5 \alpha$-THP formation from prohormones), and were subsequently infused with vehicle or FGIN 1-27 (a neurosteroidogenesis enhancer). The rats were then assessed in a behavioral battery that examined exploration (open field), anxiety (elevated plus maze), social (social interaction), and sexual (paced mating) behavior. Inhibition of $3 \alpha, 5 \alpha-$ THP formation decreased exploratory, anti-anxiety, social, and sexual behavior, as well as midbrain $3 \alpha, 5 \alpha$-THP levels. Infusions of FGIN 1-27 following $3 \alpha, 5 \alpha-$ THP inhibition restored these behaviors and midbrain $3 \alpha, 5 \alpha$-THP levels to those commensurate with control rats that had not been administered inhibitors. These findings suggest that $3 \alpha, 5 \alpha$-THP formation in the midbrain VTA may influence appetitive, as well as consummatory, aspects of mating behavior.

Reproduction (2009) 137 119-128
\end{abstract}

\section{Introduction}

Ovarian hormones, such as $17 \beta$-estradiol $\left(E_{2}\right)$ and progesterone $\left(\mathrm{P}_{4}\right)$, have coordinated actions at multiple brain sites to influence the expression of female sexual behavior in rats (typically operationalized as lordosis). In the ventromedial hypothalamus $(\mathrm{VMH}), \mathrm{E}_{2}$ and $\mathrm{P}_{4}$ have actions via cognate, intracellular progestin receptors (PRs) to initiate lordosis. In the midbrain ventral tegmental area (VTA), $\mathrm{P}_{4}$ mediates the intensity and duration of lordosis, but these effects appear to be independent of actions at PRs. Application of $\mathrm{P}_{4}$ to the VTA rapidly increases lordosis and these effects are not attenuated when $\mathrm{P}_{4}$ actions are membrane limited or when the few intracellular PRs in the VTA are blocked by antisense oligonucleotides (Frye $2001 a, 2001 b$ ). In the VTA, $\mathrm{P}_{4}$ 's actions involve formation of $5 \alpha$-pregnan- $3 \alpha$-ol-20-one $(3 \alpha, 5 \alpha$-THP), which is devoid of affinity for PRs, and has effects through $\mathrm{GABA}_{A}, \mathrm{NMDA}$, and/or dopamine-like type 1 receptors, and subsequent downstream signal transduction processes to influence lordosis (Dohi et al. 2008, Frye \& Walf 2008). Thus, progestin's actions in these regions influence the expression of female sexual behavior of rodents.

In the midbrain VTA, there are dynamic effects of progestins to mediate lordosis, and for progestin formation to be altered by female sexual behavior. The VTA richly expresses all enzymes necessary to catalyze the formation of $3 \alpha, 5 \alpha$-THP from cholesterol (Frye 2001a, $2001 b$, Mellon et al. 2001). This process of neurosteroidogenesis, which occurs in the brain independent of ovarian sources, may play an important role in progestinmediated reproductive behaviors. Neurosteroidogenesis is increased following the activation of mitochondrial benzodiazepine receptors (MBRs), which facilitate the transport of cholesterol across mitochondrial membranes (Brown \& Papadopoulos 2001). This increases the availability of cholesterol for conversion to pregnenolone via the P450 side-chain cleavage enzyme, one of the ratelimiting steps in steroid synthesis (Hall 1985, Otto et al. 1992). $3 \alpha, 5 \alpha$-THP is then formed from pregnenolone via sequential enzymatic steps involving the enzymes, $3 \beta$-hydroxysteroid dehydrogenase, $5 \alpha$-reductase, and $3 \alpha$-hydroxsteroid dehydrogenase (Plassart-Schiess \& Baulieu 2001). Prior reports have demonstrated that activating MBRs with $\mathrm{N}, \mathrm{N}$-dihexyl-2-(4-fluorophenyl)indole-3-acetamide (FGIN 1-27) enhances lordosis of rats and hamsters concomitant with increases in midbrain $3 \alpha, 5 \alpha$-THP levels (Frye \& Petralia 2003a, 2003b). Conversely, decreasing activity of MBRs in the VTA with 
1-(2-chlorophenyl)- $\mathrm{N}$-methyl- $\mathrm{N}$-(1-methylpropyl)-3-isoquinoline carboxamide (PK11195) inhibits lordosis and midbrain $3 \alpha, 5 \alpha$-THP concentrations (Frye \& Petralia $2003 a, 2003 b$ ). Naturally receptive or $\mathrm{E}_{2}$-primed rats that lack all peripheral sources of progestins have elevated levels of $3 \alpha, 5 \alpha$-THP in the midbrain VTA following mating (Frye 2001a, 2001b, Frye \& Rhodes 2006a, 2008, Frye et al. 2007). Thus, neurosteroidogenesis in the midbrain VTA may play a dynamic role in mediating female sexual behavior of rodents.

Beyond lordosis, neurosteroidogenesis in the midbrain has implications for functional effects that may be reproductively relevant. $3 \alpha, 5 \alpha$-THP and the hypothalamic-pituitary-adrenal (HPA) stress axis interact, such that $3 \alpha, 5 \alpha$-THP is altered by, and can mitigate, stress responsiveness (Engel \& Grant 2001). Exposure to extreme stressors, such as cold water swim, ether, or footshock, increases biosynthesis of pregnane and androstane neurosteroids (Purdy et al. 1991, Erskine \& Kornberg 1992, Drugan et al. 1994). Following secretion in response to a stressor, $3 \alpha, 5 \alpha$-THP can modulate HPA response to restore parasympathetic tone. In support, administration of $\mathrm{P}_{4}$ or $3 \alpha, 5 \alpha$-THP attenuates stress-induced increases in adrenocorticotropin and corticosterone, as well as transcription of CRH mRNA in the hypothalamus of stressed rats (Patchev et al. 1994, 1996). Perhaps, in part through its HPA effects, $3 \alpha, 5 \alpha$-THP can positively modulate affective behavior. $\mathrm{P}_{4}$ or $3 \alpha, 5 \alpha$-THP reduces behavioral stress responses evoked by predator odor, forced swim, or footshock (Walf \& Frye 2003, Walf et al. 2006), and can enhance antinociception, exploratory, anti-anxiety, and affiliative behavior (Finn et al. 2003, Frye \& Rhodes 2006a, 2006b, 2008, Frye et al. 2006, Engin \& Treit 2007). Given that successful mating requires suppression of fear, pain responses, and/or neophobia, rapid effects of $3 \alpha, 5 \alpha$-THP may underlie approach toward stimuli that were previously avoided.

The present study investigated the role of neurosteroidogenesis in the midbrain on appetitive (exploratory, anti-anxiety, social behavior) and consummatory (sexual) aspects of mating behaviors. We hypothesized that if biosynthesis of $3 \alpha, 5 \alpha$-THP in the midbrain VTA is important for modulating exploratory, anti-anxiety, social, and sexual behaviors, then decreasing and reinstating neurosteroidogenesis of $3 \alpha, 5 \alpha$-THP in the midbrain VTA should attenuate and enhance exploratory, anti-anxiety, social, and sexual behaviors respectively.

\section{Results}

\section{Endocrine measures}

Estrogen, progesterone, and dihydroprogesterone

There was no effect of central inhibition of $3 \alpha, 5 \alpha$-THP formation or FGIN 1-27 administration on the concentrations of plasma corticosterone or $\mathrm{E}_{2}, \mathrm{P}_{4}$, or DHP in plasma, midbrain, hippocampus, cortex, or striatum (Table 1).

\section{$3 \alpha, 5 \alpha-T H P$}

There was an interaction between $3 \alpha, 5 \alpha$-THP inhibitors and FGIN 1-27 administration on midbrain $3 \alpha, 5 \alpha-$ THP levels $(F(3,67)=4.71, P<0.05)$, which was due to an increase in $3 \alpha, 5 \alpha$-THP levels following FGIN 1-27 administration in rats administered PK11195 and/or indomethacin, but not vehicle (Fig. 1, top). Plasma $3 \alpha, 5 \alpha$-THP levels were not altered by $3 \alpha, 5 \alpha$-THP inhibition in the brain or FGIN 1-27 infusion (Fig. 1, bottom).

\section{Behavioral measures}

\section{Open field}

Inhibition of $3 \alpha, 5 \alpha$-THP significantly decreased central entries in the open field $(F(3,67)=2.70, P=0.05)$. Indomethacin, alone or in conjunction with PK11195, significantly reduced central square entries. Midbrain levels of $3 \alpha, 5 \alpha$-THP also significantly predicted central entries $(t(69)=2.96, P<0.05)$ and accounted for a significant proportion of central entry variance $\left(R^{2}=0.11, F(1,69)=8.74, P<0.05\right)$.

There was no effect of inhibition of $3 \alpha, 5 \alpha$-THP or FGIN 1-27 administration on peripheral or total entries in the open field (Table 2).

\section{Elevated plus maze}

There were significant interactions between inhibition of $3 \alpha, 5 \alpha$-THP formation and FGIN 1-27 administration on the time spent on the open $(F(3,67)=2.78, P<0.05)$ and closed $(F(3,67)=5.50, P<0.05)$ arms of the elevated plus maze. This was due to increased time spent on the open arms of the plus maze (Fig. 2, top), and decreased time spent on the closed arms (Table 2), among rats administered inhibitor followed by infusions of FGIN 1-27 but not vehicle. Midbrain levels of $3 \alpha, 5 \alpha$-THP also significantly predicted open arm time $(t(69)=2.82, P<0.05)$ and accounted for a significant proportion of open arm time variance $\left(R^{2}=0.10, F(1,69)=7.97, P<0.05\right)$.

There was a main effect for rats receiving infusions of inhibitor to make fewer open arm entries in the elevated plus maze than those administered vehicle or FGIN 1-27 without inhibitor infusions $(F(3,67)=5.70, P<0.05$; Table 2). No effects of central manipulation were observed for closed or total arm entries (Table 2).

\section{Social interaction}

Although there was an apparent effect of indomethacin, with or without PK11195, to decrease the duration of time spent in social interaction with a conspecific, this effect was not significant. Administration of FGIN 1-27 increased social interaction of rats, overall $(F(1,69)=3.80, P<0.05$; Fig. 2, bottom). Midbrain levels of $3 \alpha, 5 \alpha$-THP also significantly predicted the time spent in social interaction $(t(69)=2.45, P<0.05)$ and accounted for a significant 
Table 1 Neuroendocrine measures of female rats infused with vehicle, PK11195, and/or indomethacin with or without subsequent infusions of vehicle or FGIN 1-27 to the ventral tegmental area of the midbrain (mean \pm s.E.M.).

\begin{tabular}{|c|c|c|c|c|c|c|c|c|}
\hline $\begin{array}{l}\text { Infusate } \# 1 \\
\text { Infusate } \# 2 \\
\text { Infusate } \# 3\end{array}$ & $\begin{array}{l}\text { Vehicle } \\
\text { Vehicle } \\
\text { Vehicle }\end{array}$ & $\begin{array}{l}\text { Vehicle } \\
\text { Vehicle } \\
\text { FGIN 1-27 }\end{array}$ & $\begin{array}{l}\text { PK11195 } \\
\text { Vehicle } \\
\text { Vehicle }\end{array}$ & $\begin{array}{l}\text { PK11195 } \\
\text { Vehicle } \\
\text { FGIN 1-27 }\end{array}$ & $\begin{array}{l}\text { Vehicle } \\
\text { Indomethacin } \\
\text { Vehicle }\end{array}$ & $\begin{array}{l}\text { Vehicle } \\
\text { Indomethacin } \\
\text { FGIN 1-27 }\end{array}$ & $\begin{array}{l}\text { PK11195 } \\
\text { Indomethacin } \\
\text { Vehicle }\end{array}$ & $\begin{array}{l}\text { PK11195 } \\
\text { Indomethacin } \\
\text { FGIN 1-27 }\end{array}$ \\
\hline \multicolumn{9}{|l|}{ Corticosterone } \\
\hline \multicolumn{9}{|l|}{$\mathrm{E}_{2}^{\mathrm{a}}$} \\
\hline Serum (pg/ml) & $18.3 \pm 3.3$ & $13.2 \pm 3.1$ & $10.3 \pm 2.4$ & $14.6 \pm 3.6$ & $11.6 \pm 2.8$ & $14.3 \pm 2.9$ & $13.2 \pm 2.9$ & $12.7 \pm 3.4$ \\
\hline Midbrain (pg/g) & $1.4 \pm 0.1$ & $1.2 \pm 0.4$ & $1.4 \pm 0.2$ & $1.4 \pm 0.2$ & $1.4 \pm 0.2$ & $1.6 \pm 0.2$ & $1.1 \pm 0.2$ & $1.4 \pm 0.2$ \\
\hline Hippocampus (pg/g) & $1.3 \pm 0.3$ & $1.0 \pm 0.3$ & $1.8 \pm 0.3$ & $1.6 \pm 0.3$ & $1.4 \pm 0.7$ & $1.4 \pm 0.4$ & $0.7 \pm 0.2$ & $1.7 \pm 0.2$ \\
\hline Cortex (pg/g) & $1.5 \pm 0.3$ & $1.3 \pm 0.4$ & $1.8 \pm 0.3$ & $1.6 \pm 0.3$ & $1.3 \pm 0.3$ & $1.5 \pm 0.4$ & $1.4 \pm 0.3$ & $1.8 \pm 0.3$ \\
\hline Diencephalon (pg/g) & $1.1 \pm 0.2$ & $1.2 \pm 0.3$ & $1.3 \pm 0.3$ & $1.5 \pm 0.3$ & $1.7 \pm 0.3$ & $1.4 \pm 0.4$ & $1.4 \pm 0.3$ & $1.8 \pm 0.5$ \\
\hline Interbrain (pg/g) & $0.7 \pm 0.2$ & $1.3 \pm 0.2$ & $0.9 \pm 0.2$ & $1.3 \pm 0.3$ & $1.0 \pm 0.2$ & $0.7 \pm 0.2$ & $0.9 \pm 0.2$ & $1.1 \pm 0.1$ \\
\hline \multicolumn{9}{|l|}{$\mathrm{P}_{4}^{\mathrm{b}}$} \\
\hline Serum (ng/ml) & $19.6 \pm 2.9$ & $14.3 \pm 3.6$ & $17.1 \pm 2.6$ & $17.1 \pm 4.0$ & $14.6 \pm 3.4$ & $19.6 \pm 3.7$ & $15.0 \pm 3.5$ & $17.4 \pm 4.5$ \\
\hline Midbrain (ng/g) & $1.9 \pm 0.4$ & $1.4 \pm 0.4$ & $1.4 \pm 0.5$ & $1.5 \pm 0.5$ & $1.8 \pm 0.3$ & $1.4 \pm 0.4$ & $1.2 \pm 0.3$ & $1.4 \pm 0.4$ \\
\hline Hippocampus (ng/g) & $2.3 \pm 0.4$ & $1.5 \pm 0.5$ & $1.4 \pm 0.4$ & $1.9 \pm 0.4$ & $2.4 \pm 0.8$ & $2.9 \pm 0.4$ & $1.8 \pm 0.4$ & $1.9 \pm 0.5$ \\
\hline Cortex $(\mathrm{ng} / \mathrm{g})$ & $1.8 \pm 0.5$ & $1.8 \pm 0.7$ & $2.9 \pm 0.7$ & $2.9 \pm 0.8$ & $2.7 \pm 0.4$ & $2.8 \pm 0.4$ & $2.5 \pm 0.4$ & $2.4 \pm 0.6$ \\
\hline Diencephalon (ng/g) & $1.4 \pm 0.4$ & $1.3 \pm 0.5$ & $1.9 \pm 0.2$ & $1.9 \pm 0.3$ & $1.9 \pm 0.3$ & $2.0 \pm 0.2$ & $2.1 \pm 0.2$ & $1.7 \pm 0.4$ \\
\hline Interbrain (ng/g) & $1.4 \pm 0.4$ & $1.3 \pm 0.5$ & $1.7 \pm 0.4$ & $1.7 \pm 0.3$ & $2.1 \pm 0.5$ & $2.1 \pm 0.4$ & $1.5 \pm 0.5$ & $1.3 \pm 0.3$ \\
\hline \multicolumn{9}{|l|}{$\mathrm{DHP}$} \\
\hline Serum (ng/ml) & $20.4 \pm 4.0$ & $14.6 \pm 5.2$ & $10.5 \pm 4.5$ & $10.4 \pm 4.4$ & $16.7 \pm 3.6$ & $15.1 \pm 5.0$ & $14.5 \pm 4.2$ & $19.9 \pm 3.4$ \\
\hline Midbrain (ng/g) & $8.0 \pm 0.6$ & $6.7 \pm 1.2$ & $6.3 \pm 1.0$ & $7.2 \pm 1.1$ & $8.3 \pm 1.0$ & $7.2 \pm 1.2$ & $5.7 \pm 0.4$ & $7.2 \pm 1.2$ \\
\hline Hippocampus (ng/g) & $10.7 \pm 0.7$ & $7.7 \pm 1.7$ & $7.2 \pm 1.6$ & $9.6 \pm 1.4$ & $10.0 \pm 4.0$ & $8.0 \pm 2.3$ & $7.5 \pm 0.8$ & $6.9 \pm 2.0$ \\
\hline Cortex $(\mathrm{ng} / \mathrm{g})$ & $4.6 \pm 0.4$ & $4.7 \pm 0.7$ & $3.9 \pm 1.0$ & $4.8 \pm 0.8$ & $3.5 \pm 0.6$ & $4.2 \pm 0.7$ & $4.5 \pm 0.6$ & $5.1 \pm 1.0$ \\
\hline Diencephalon (ng/g) & $4.6 \pm 0.3$ & $4.3 \pm 0.3$ & $3.5 \pm 0.6$ & $4.4 \pm 0.7$ & $4.2 \pm 0.6$ & $4.7 \pm 0.4$ & $3.9 \pm 0.2$ & $4.8 \pm 0.9$ \\
\hline Interbrain (ng/g) & $1.4 \pm 0.1$ & $1.5 \pm 0.1$ & $1.1 \pm 0.2$ & $1.2 \pm 0.1$ & $1.2 \pm 0.2$ & $1.3 \pm 0.2$ & $1.2 \pm 0.2$ & $1.0 \pm 0.2$ \\
\hline \multicolumn{9}{|l|}{$3 \alpha, 5 \alpha-\mathrm{THP}$} \\
\hline Serum (ng/ml) & $17.4 \pm 7.2$ & $12.4 \pm 8.1$ & $21.4 \pm 8.8$ & $31.6 \pm 10.2$ & $25.5 \pm 9.1$ & $27.5 \pm 10.9$ & $24.8 \pm 9.0$ & $19.1 \pm 9.4$ \\
\hline Hippocampus (ng/g) & $18.4 \pm 8.0$ & $12.5 \pm 5.9$ & $13.3 \pm 7.1$ & $19.6 \pm 7.8$ & $20.0 \pm 6.5$ & $27.0 \pm 9.8$ & $20.2 \pm 6.1$ & $21.9 \pm 6.8$ \\
\hline Cortex (ng/g) & $8.4 \pm 1.6$ & $6.4 \pm 2.6$ & $3.4 \pm 1.5$ & $6.0 \pm 2.4$ & $5.2 \pm 1.2$ & $5.1 \pm 1.9$ & $4.9 \pm 1.7$ & $5.3 \pm 1.9$ \\
\hline Diencephalon (ng/g) & $5.9 \pm 1.7$ & $4.8 \pm 1.8$ & $2.4 \pm 1.3$ & $4.2 \pm 1.7$ & $5.8 \pm 1.6$ & $4.9 \pm 1.8$ & $4.5 \pm 1.3$ & $6.5 \pm 2.1$ \\
\hline Interbrain (ng/g) & $5.5 \pm 2.9$ & $5.8 \pm 2.1$ & $4.1 \pm 1.7$ & $5.8 \pm 2.3$ & $5.8 \pm 2.2$ & $4.6 \pm 2.4$ & $4.4 \pm 2.0$ & $2.3 \pm 1.6$ \\
\hline
\end{tabular}

${ }^{\mathrm{a}}$ Estrogen. ${ }^{\mathrm{b}}$ Progesterone. ${ }^{\mathrm{c}}$ Dihydroprogesterone.

proportion of social interaction variance $\left(R^{2}=0.08\right.$, $F(1,69)=6.01, P<0.05)$.

\section{Sex behavior}

There was a significant interaction between $3 \alpha, 5 \alpha$-THP inhibition and FGIN 1-27 administration on lordosis quotients $(F(3,67)=3.18, \quad P<0.05$; Fig. 3 , top $)$ and lordosis ratings $(F(3,67)=3.34, P<0.05$; Fig. 3 , bottom). These interactions were due to FGIN 1-27, but not vehicle, administration increasing lordosis quotients and ratings of rats infused with inhibitors. Midbrain levels of $3 \alpha, 5 \alpha$-THP also significantly predicted lordosis quotient $(t(69)=3.74, P<0.05)$ and lordosis rating $(t(69)=4.05, P<0.05)$ and accounted for a significant proportion of variance in lordosis quotients $\left(R^{2}=0.17\right.$, $F(1,69)=14.00, P<0.05)$ and lordosis ratings $\left(R^{2}=0.19\right.$, $F(1,69)=16.43, P<0.05)$.

FGIN 1-27 administration also significantly interacted with inhibitor condition on the percentage of proceptive behaviors displayed per sexual contact $(F(3,67)=3.53$, $P<0.05)$. FGIN 1-27 infusions following inhibitors increased proceptivity quotients among vehicle- and PK11195-infused rats, but not among the rats infused with indomethacin alone or in conjunction with PK11195 (Fig. 4, top). Vehicle infusions following inhibitors did not alter proceptivity and proceptivity could not be predicted by midbrain $3 \alpha, 5 \alpha$-THP levels.

Percentage of aggression in response to sexual contacts appeared to be the greatest among indomethacin-infused rats; however, this was not significant (Fig. 4, bottom). Percentage of exits from the male to the neutral compartment of the paced mating chamber also appeared to decrease among females infused with indomethacin, with or without PK11195, but this was not significant (Table 3). Neither aggression quotients nor percentage of exits was predicted by midbrain $3 \alpha, 5 \alpha$-THP levels.

\section{Discussion}

The results of the present study supported our hypothesis that manipulating neurosteroidogenesis of $3 \alpha, 5 \alpha-\mathrm{THP}$ in the midbrain VTA would alter exploratory, anti-anxiety, and reproductive behavior of female rats. First, administration of PK11195, either alone or in conjunction with indomethacin, decreased central square entries in the open field (albeit this did not reach statistical significance), decreased time spent on the open arms of the plus maze, and decreased lordosis quotients and ratings concomitant with decreased levels of $3 \alpha, 5 \alpha$-THP in the midbrain compared with vehicle administration. Second, FGIN 1 27 administration increased time spent on the open arms of 


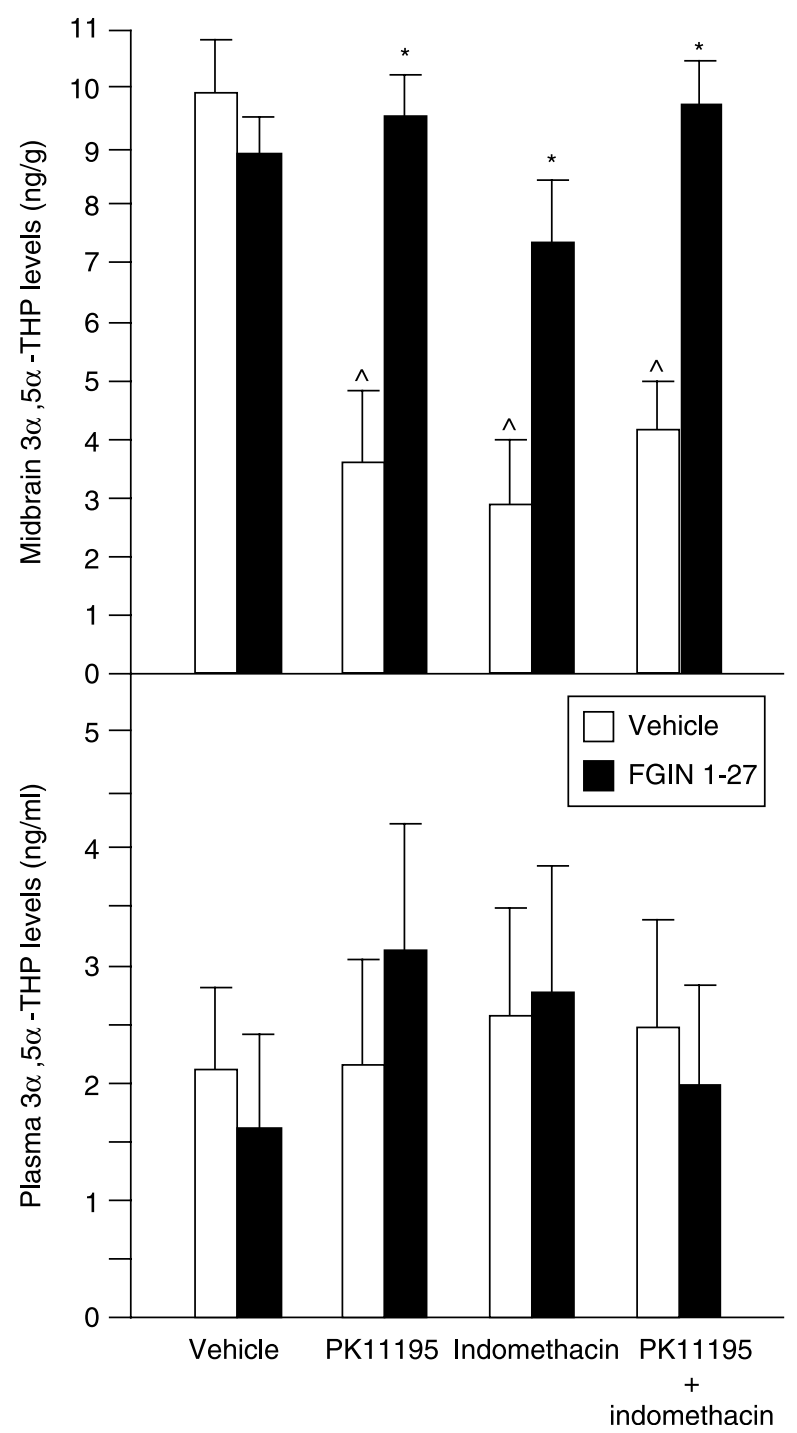

Figure 1 Midbrain (top) and plasma (bottom) $3 \alpha, 5 \alpha$-THP levels (mean + S.E.M.) among rats infused with vehicle, PK11195, and/or indomethacin with or without subsequent infusions of vehicle or FGIN 1-27. $\wedge$ Significant decrease among inhibitor-infused rats compared with rats infused only with vehicle $(P<0.05)$. *Significant enhancement following FGIN 1-27 compared with infusion of inhibitor followed by vehicle infusions $(P<0.05)$.

the elevated plus maze, time spent in social interaction, and enhanced lordosis as well as increasing levels of $3 \alpha, 5 \alpha-$ THP in the midbrain VTA. Together, these data suggest that neurosteroidogenesis of $3 \alpha, 5 \alpha$-THP in the midbrain VTA is an important factor for modulating lordosis and other reproductively relevant behaviors.

The present findings confirm previous reports that manipulating neurosteroidogenesis can alter female sexual behavior. In support, blocking P450 side-chain cleavage decreased proceptive behaviors of ovariectomized/adrenalectomized $\mathrm{E}_{2}$-primed rats (Micevych et al. 2007). Furthermore, administration of PK11195 or FGIN 1-27 to the midbrain VTA respectively decreases and
Table 2 Motor behavior in open field and elevated plus maze tasks of female rats infused with vehicle, PK11195, and/or indomethacin with or without subsequent infusions of vehicle or FGIN 1-27 (mean \pm s.E.M.).

\begin{tabular}{|c|c|c|c|c|}
\hline & $\begin{array}{l}\text { Vehicle/ } \\
\text { vehicle }\end{array}$ & $\begin{array}{l}\text { PK11195/ } \\
\text { vehicle }\end{array}$ & $\begin{array}{c}\text { Vehicle/ } \\
\text { indomethacin }\end{array}$ & $\begin{array}{c}\text { PK11195/ } \\
\text { indomethacin }\end{array}$ \\
\hline \multicolumn{5}{|c|}{ Total entries in open field } \\
\hline Vehicle & $382 \pm 31$ & $271 \pm 42$ & $329 \pm 41$ & $255 \pm 51$ \\
\hline FGIN 1-27 & $357 \pm 27$ & $344 \pm 20$ & $329 \pm 27$ & $369 \pm 52$ \\
\hline \multicolumn{5}{|c|}{ Peripheral entries in open field } \\
\hline Vehicle & $265 \pm-23$ & $199 \pm 31$ & $253 \pm 23$ & $195 \pm 27$ \\
\hline FGIN 1-27 & $246 \pm 13$ & $241 \pm 11$ & $261 \pm 28$ & $272 \pm 33$ \\
\hline \multicolumn{5}{|c|}{ Central entries in open field* } \\
\hline Vehicle & $117 \pm 15$ & $72 \pm 14$ & $76 \pm 22^{*}$ & $61 \pm 16^{*}$ \\
\hline FGIN 1-27 & $111 \pm 12$ & $102 \pm 13$ & $68 \pm 10$ & $97 \pm 22$ \\
\hline \multicolumn{5}{|c|}{ Closed arm time in elevated plus maze ${ }^{+}$} \\
\hline Vehicle & $111 \pm 13$ & $222 \pm 18$ & $226 \pm 16^{+}$ & $227 \pm 11^{\dagger}$ \\
\hline FGIN 1-27 & $149 \pm 26$ & $121 \pm 15$ & $178 \pm 19$ & $165 \pm 23$ \\
\hline \multicolumn{5}{|c|}{ Total arm entries in elevated plus maze } \\
\hline Vehicle & $20 \pm 3$ & $13 \pm 3$ & $12 \pm 3$ & $12 \pm 1$ \\
\hline FGIN 1-27 & $17 \pm 1$ & $16 \pm 2$ & $12 \pm 2$ & $14 \pm 2$ \\
\hline \multicolumn{5}{|c|}{ Open arm entries in elevated plus maze* } \\
\hline Vehicle & $10 \pm 2$ & $4 \pm 1^{*}$ & $5 \pm 1^{*}$ & $4 \pm 1^{*}$ \\
\hline FGIN 1-27 & $8 \pm 1$ & $6 \pm 1$ & $5 \pm 1$ & $6 \pm 1$ \\
\hline \multicolumn{5}{|c|}{ Closed arm entries in elevated plus maze } \\
\hline Vehicle & $10 \pm 2$ & $8 \pm 2$ & $8 \pm 1$ & $8 \pm 1$ \\
\hline FGIN 1-27 & $9 \pm 1$ & $10 \pm 1$ & $8 \pm 1$ & $8 \pm 1$ \\
\hline
\end{tabular}

*Significant main effect for inhibitor-infused groups to make fewer entries than vehicle/vehicle-infused groups $(P<0.05)$. ${ }^{\dagger}$ Significant interaction for FGIN 1-27 infusions to decrease closed arm time among inhibitor-infused, but not vehicle/vehicle-infused, rats $(P<0.05)$.

increases lordosis of rats and hamsters concomitant with changes in the midbrain VTA levels of $3 \alpha, 5 \alpha$-THP (Frye \& Petralia 2003a, 2003b). Similarly, the present study demonstrates that decreasing $3 \alpha, 5 \alpha$-THP levels in the midbrain VTA with the administration of PK11195 results in decreased expression and quality of lordosis. We have extended these prior data to show that administration of FGIN 1-27 can overcome the effects of PK11195 on 3 $\alpha, 5 \alpha$ THP concentrations and lordosis. Administration of FGIN 1-27 attenuated deficits in lordosis induced by PK11195, such that lordosis was comparable with that of vehicleadministered rats. Thus, neurosteroidogenesis of $3 \alpha, 5 \alpha-$ THP appears to play a critical role in mediating sexual behavior of female rats.

The present results also extend previous reports to suggest that neurosteroidogenesis of $3 \alpha, 5 \alpha$-THP in the midbrain VTA can modulate other reproductively relevant behaviors. Prior reports have shown that increasing neurosteroidogenesis in the hippocampus with FGIN 1-27 enhances anti-anxiety behavior in the elevated plus maze (Bitran et al. 2000). In the present experiment, PK11195 decreased midbrain levels of $3 \alpha, 5 \alpha$-THP and decreased exploratory and anti-anxiety behaviors while increasing $3 \alpha, 5 \alpha$-THP levels in the midbrain with FGIN 1-27 attenuated the effects of PK11195 on anti-anxiety behavior. Interestingly, social behavior was seemingly unaffected by decreasing activation of MBRs, but was enhanced following stimulation of MBRs. These data suggest that neurosteroidogenesis in the midbrain VTA 


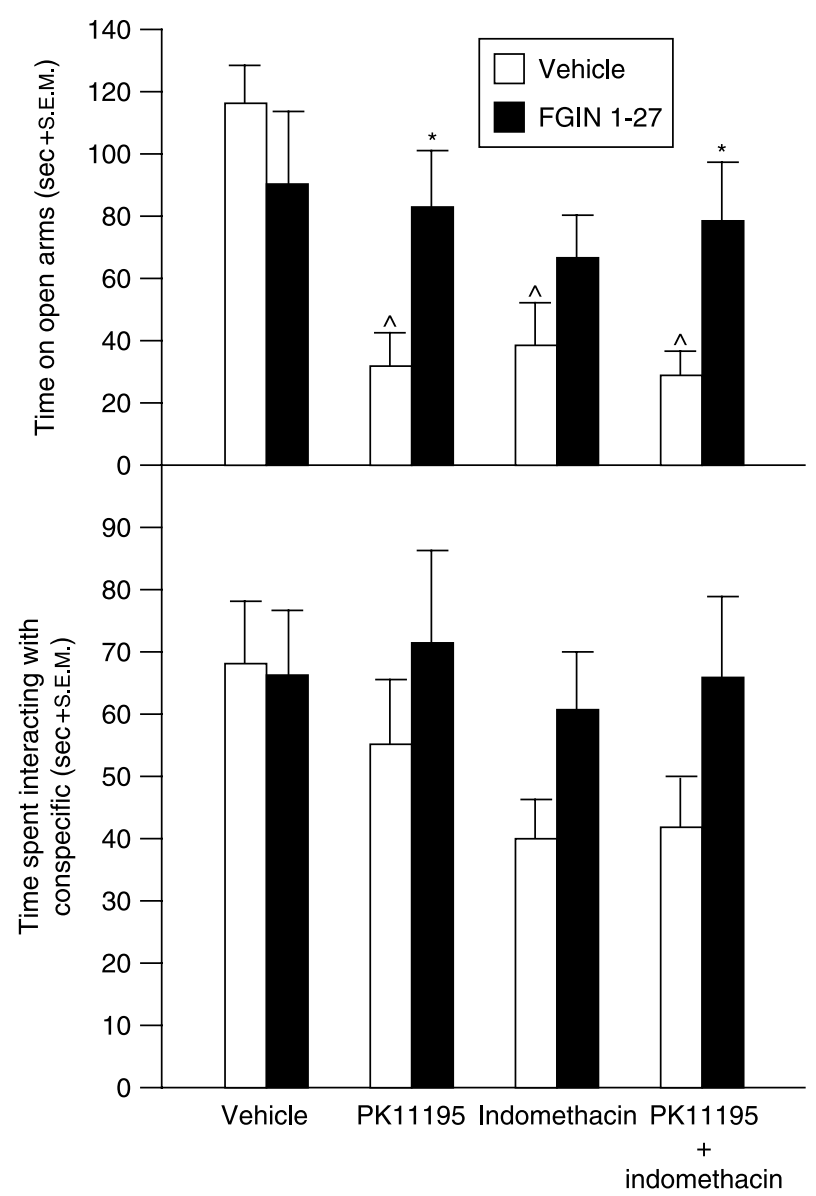

Figure 2 Time (s) spent on the open arms of an elevated plus maze (top, mean + S.E.M.) and time (s) spent interacting with a conspecific (bottom, mean + S.E.M.) among rats infused with vehicle, PK11195, and/or indomethacin with or without subsequent infusions of vehicle or FGIN 1-27. ^Significant decrease among inhibitor-infused rats compared with rats infused only with vehicle $(P<0.05)$. *Significant enhancement following FGIN 1-27 compared with infusion of inhibitor followed by vehicle infusions $(P<0.05)$.

may play an important role in modulating behaviors beyond lordosis, and that there may be different effects of manipulating neurosteroidogenesis depending upon behaviors examined.

The results of the present study are intriguing as they suggest that rapid formation of $3 \alpha, 5 \alpha-$ THP in the midbrain VTA may be critically important for mediating consummatory reproductive behaviors, as well as appetitive reproductive behaviors important for successful initiation of mating (exploration, anti-anxiety, social interaction). Of note, the effects of manipulations of neurosteroidogenesis in the midbrain VTA were not consistent across all behaviors examined, suggesting that other neuroendocrine factors may also play an important role. This notion is further supported by evidence from the regression analyses that revealed that $3 \alpha, 5 \alpha$-THP midbrain levels explained between 8 and $19 \%$ of the variance in most behaviors studied. $3 \alpha, 5 \alpha$-THP accounted for most variance in sexual

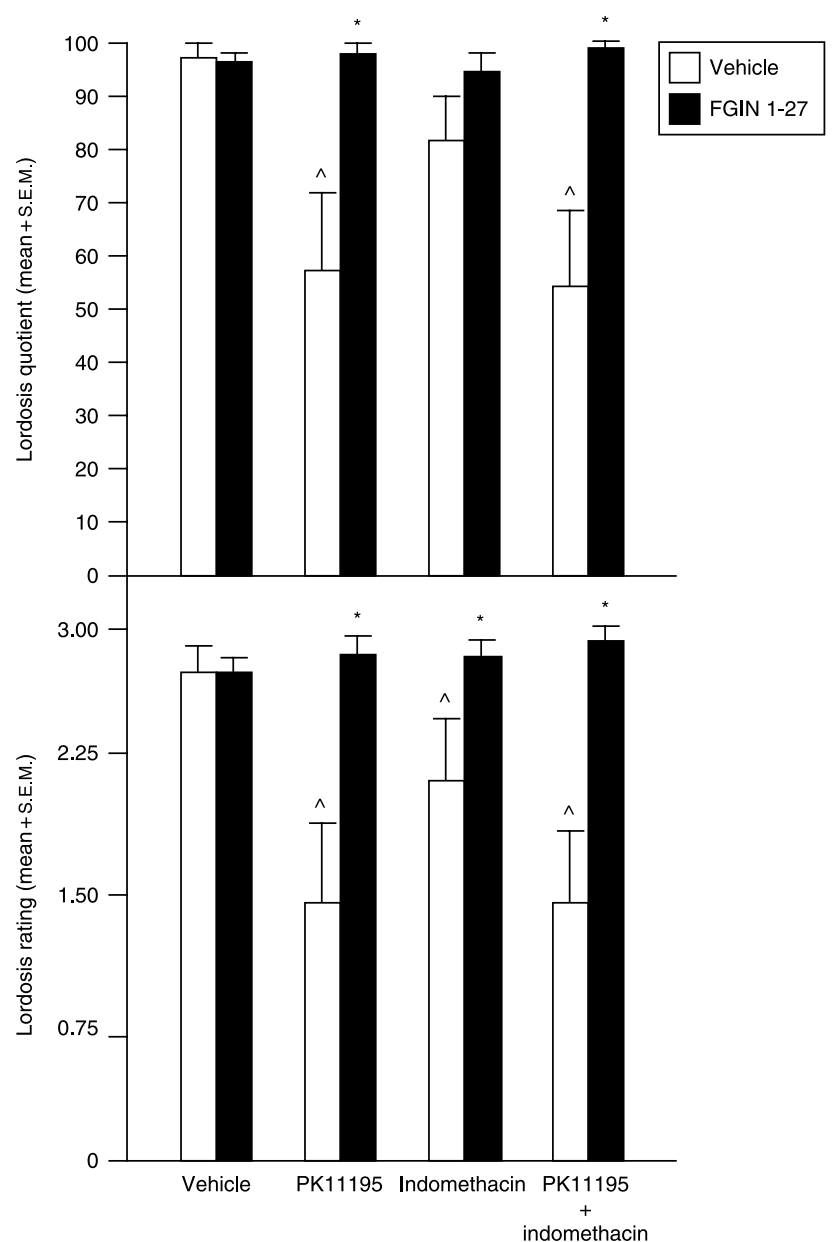

Figure 3 Percentage (top) and intensity (bottom) of lordosis (mean + S.E.M.) among sexually contacted rats infused with vehicle, PK11195, and/or indomethacin with or without subsequent infusions of vehicle or FGIN 1-27. ^Significant decrease among inhibitor-infused rats compared with rats infused only with vehicle $(P<0.05)$. *Significant enhancement following FGIN 1-27 compared with infusion of inhibitor followed by vehicle infusions $(P<0.05)$.

behavior as opposed to appetitive behaviors examined, which may be, in part, due to the fact that all subjects in the present study engaged in paced mating prior to tissue collection, which enhances $3 \alpha, 5 \alpha$-THP concentrations in the midbrain (Frye \& Rhodes 2006b). Enhancement of $3 \alpha, 5 \alpha$-THP via FGIN 1-27 appeared to increase exploratory, anti-anxiety, social, and sexual behavior overall, but inhibition of $3 \alpha, 5 \alpha$-THP did not necessarily attenuate these behaviors to the same degree. Exploration in the open field and social interaction were modestly decreased by inhibitors but social interaction was significantly enhanced by FGIN 1-27 infusion, whereas only after PK11195 did FGIN 1-27 reinstate exploratory behavior in the open field. These results of inhibitors on behavior further suggest that FGIN 1-27 most readily reinstated neurosteroidogenesisrelated decrement via PK11195 compared with infusions that included indomethacin. This was most saliently observed on the measure of proceptivity wherein there 


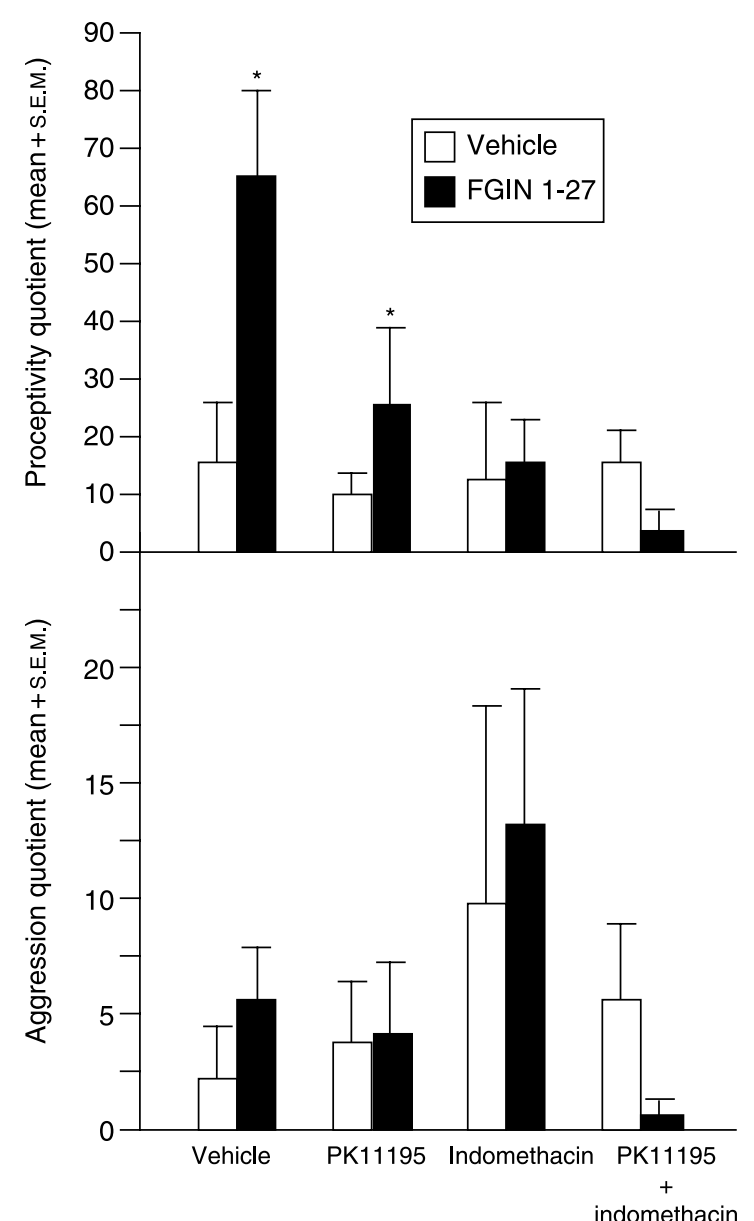

Figure 4 Percentage of proceptive (top; hopping, darting, ear wiggling) and aggressive behaviors (bottom; vocalizing, threatening, attacking) in response to sexual contact (mean + S.E.M.) among rats infused with vehicle, PK11195, and/or indomethacin with or without subsequent infusions of vehicle or FGIN 1-27. *Significant enhancement following FGIN 1-27 compared with infusion of inhibitor followed by vehicle infusions $(P<0.05)$

was a stair-step effect for FGIN 1-27 to enhance proceptivity greatly among the rats receiving only vehicle infusions, slightly less among the rats infused only with PK11195, and not at all among indomethacin-infused rats. Together, these findings suggest that exploratory, antianxiety, and sexual behavior may be particularly sensitive to the effects of neurosteroidogenesis.

In the present study, we examined the effects of manipulating neurosteroidogenesis in the midbrain VTA. While $3 \alpha, 5 \alpha$-THP was found to be enhanced in this investigation, recent findings suggest that progesterone can also have actions at novel membrane-bound receptors (Dohi et al. 2008). That attenuation of $3 \alpha, 5 \alpha-$ THP formation in the present study reduced appetitive and consummatory sexual behaviors, yet $3 \alpha, 5 \alpha$-THP content accounted for, at most, $19 \%$ of variance in behavior, may suggest that $3 \alpha, 5 \alpha$-THP plays a critical role underlying the maintenance of other novel
Table 3 Percentage of pacing behavior displayed in a paced mating task by female rats infused with vehicle, PK11195, and/or indomethacin with or without subsequent infusions of vehicle or FGIN 1-27 (mean \pm S.E.M.).

\begin{tabular}{lcccc}
\hline & Vehicle/ & Vehicle/ \\
vehicle $(\%)$ & $\begin{array}{c}\text { PK11195/ } \\
\text { vehicle }(\%)\end{array}$ & $\begin{array}{c}\text { PK1195/ } \\
\text { indomethacin } \\
(\%)\end{array}$ & $\begin{array}{c}\text { indomethacin } \\
(\%)\end{array}$ \\
\hline $\begin{array}{l}\text { Percentage of pacing in paced mating } \\
\text { Vehicle }\end{array}$ & $9 \pm 6$ & $10 \pm 5$ & $4 \pm 2$ & $6 \pm 3$ \\
FGIN 1-27 & $8 \pm 3$ & $9 \pm 5$ & $5 \pm 3$ & $1 \pm 1$ \\
\hline
\end{tabular}

neuroendocrine substrates that are important for these behaviors. Distribution and functional effects of novel substrates and targets of action are not yet clear but pose intriguing new mechanisms for future study. Given the effects on various aspects of appetitive behavior that were revealed, future investigations will focus on the role of neurosteroidogenesis in other CNS sites known to be important for mediating exploratory, anti-anxiety, social, and sexual behaviors, as well as novel progestin targets.

\section{Materials and Methods}

These methods were pre-approved by the Institutional Animal Care and Use Committee at The University at Albany-SUNY.

\section{Animals}

Adult, intact, Long-Evans female rats $(n=71)$ were bred in the Life Sciences Laboratory Animal Care Facilities at The University at Albany from the stock obtained from Charles River (Germantown, NY, USA). Rats were group housed in polycarbonate cages $(45 \times 24 \times 21 \mathrm{~cm})$ in a temperaturecontrolled room $\left(21 \pm 1^{\circ} \mathrm{C}\right)$ in the Laboratory Animal Care Facility. The rats were maintained on a 12:12 $\mathrm{h}$ reversed light cycle (lights off $0800 \mathrm{~h}$ ) with continuous free access to Purina Rat Chow and tap water in their home cages.

\section{Surgery}

Rats were stereotaxically implanted with bilateral guide cannulae aimed at the medial aspect of the VTA (from bregma: $\mathrm{AP}=-5.3, \mathrm{ML}= \pm 0.4, \mathrm{DV}=-7.0)$ under xylazine $(12 \mathrm{mg} / \mathrm{kg})$ and ketamine $(70 \mathrm{mg} / \mathrm{kg}$ ) anesthesia. Guide cannulae consisted of modified 23 gauge thin wall stainless steel needles with 30 gauge removable inserts. Following surgery, animals were monitored for loss of weight, righting response, flank stimulation response, and/or muscle tone (Marshall \& Teitelbaum 1974). No rats failed these assessments.

\section{Hormonal milieu}

\section{Endogenous}

Vaginal epithelium was examined daily (between $0700 \mathrm{~h}$ and $0800 \mathrm{~h}$ ) to determine the phase of the estrous cycle, as per previous methods (Long \& Evans 1922, Frye et al. 2000). Rats 
were cycled through two normal estrous cycles (4- to 5-day cycle) prior to testing. The rats were tested on the evening of proestrus, when $E_{2}$ levels are declining, but progestin levels are high relative to other phases of the estrous cycle (Feder 1984, Frye \& Bayon 1999).

\section{Exogenous}

Rats received bilateral infusions of either indomethacin $(10 \mu \mathrm{g} / \mu \mathrm{l}, 10-\mathrm{min}$ incubation), PK11195 (400 ng/ $\mu \mathrm{l}, 20-\mathrm{min}$ incubation), or an equal volume of $\beta$-cyclodextrin vehicle to the VTA. We have found that this dose of indomethacin is sufficient to attenuate lordosis when infused to VTA of naturally receptive or hormone-primed rats or hamsters (Frye \& Vongher 2001). Dosing of PK11195 was based on the past findings that identified $400 \mathrm{ng}$ as the minimum dose needed to reliably attenuate lordosis among sexually receptive female rats in a dose-response regimen (Bitran et al. 2000, Frye \& Petralia 2003a, 2003b). Following the first infusion, the rats that received PK11195 next received infusions of indomethacin while rats in other groups received a second infusion of $\beta$-cyclodextrin vehicle. Ten minutes later, the rats received infusions of FGIN 1-27 $(5 \mu \mathrm{g} / \mu \mathrm{l})$ or an equal volume of saline vehicle. This dosage of FGIN 1-27 is sufficient to enhance lordosis of estrogen- and progesterone-primed ovariectomized rats to the levels that are commensurate with naturally, sexually receptive rats, compared with lower or higher doses (Bitran et al. 2000, Frye \& Petralia 2003a, 2003b). These manipulations yielded the following eight groups: vehicle/ vehicle/vehicle $(n=10)$, vehicle/vehicle/FGIN 1-27 $(n=8)$, PK11195/vehicle/vehicle ( $n=9)$, PK11195/vehicle/FGIN 1-27 $(n=9)$, vehicle/indomethacin/vehicle $(n=8)$, vehicle/indomethacin/FGIN 1-27 $(n=9)$, PK11195/indomethacin/vehicle $(n=10)$, and PK11195/indomethacin/FGIN 1-27 $(n=8)$. Central infusions were administered at a rate of $1 \mu \mathrm{l} / \mathrm{min}$ through a 30 gauge needle attached to PE-20 tubing and a $5 \mu \mathrm{l}$ Hamilton syringe. The infusion needle was left in place for $60 \mathrm{~s}$ following infusions to reduce possible displacement of infusate. The rats were tested in the behavioral battery described below.

\section{Behavioral testing}

All rats were tested individually through the following test battery of exploratory, anxiety, social, and sexual behavior in the order described below. All testing occurred in a single room with testing apparatus brightly lit from above with three fluorescent bulbs (32 W each). The rats were tested sequentially through tasks, with no breaks between individual tasks, except the time required to clean the apparatus and transition rats from one task to another. As such, assessment in the entire battery took about 45-50 min for each rat. We have utilized these behavioral measures in the past as individual tasks (Frye et al. 2007), small batteries of anxiety, social, or sexual measures only (Frye et al. 2007), or as a single battery of testing (Frye \& Rhodes 2006b). We find that behavioral and neuroendocrine status is not significantly affected by exposure to any task other than mating, which is the last task in the battery described below (Frye et al. 2007).
Behavioral data were collected with the ANY-Maze data collection program (Stoelting Co., Wheat Dale, IL, USA), and by an observer blind to the condition of experimental rats and the hypothesized outcome of the study. There was a 97\% concordance rate between data that were collected by ANY-Maze and that collected by the uninformed observer. As such, the data collected via ANY-Maze were utilized in behavioral analyses.

\section{Open field}

Behavior in the open field is used as a measure of exploration, anxiety, and locomotor behavior (Blizard et al. 1975, Frye et al. 2000). The open field $(76 \times 57 \times 35 \mathrm{~cm})$ has a 48 -square grid floor ( $6 \times 8$ squares, $9.5 \mathrm{~cm} / \mathrm{side})$ : there is an overhead light illuminating the central squares (all but the 24 perimeter squares were considered central). As per previous methods, rats were placed in the open field and the path of their exploration was recorded for $5 \mathrm{~min}$. The number of squares entered by rats in the center or periphery of the grid was calculated and these data were added together to yield the total number of squares entered. Prior reports indicate that total square entries in this task are robustly modulated by hormonal status of female rats and by steroid sensitive manipulations (Birke \& Archer 1975, Walf \& Frye 2007, Frye \& Rhodes 2008). Other motor measures, such as rearing, may not be so sensitive to manipulations that alter hormonal milieu (Avitsur et al. 1995, Renard et al. 2007, Liang et al. 2008). Since the present study utilized a sample of female rats that were all matched on the phase of estrous cycle, motor differences were expected to be minimized. Thus, central square entries were utilized as an index of anti-anxiety, and total square entries as an index of thigmotaxis and motor behavior.

\section{Elevated plus maze}

Behavior in the elevated plus maze is also utilized to assess exploration, anxiety, and motor behavior (File 1990, Frye et al. 2000). The elevated plus maze consists of four arms, $49 \mathrm{~cm}$ long and $10 \mathrm{~cm}$ wide, elevated $50 \mathrm{~cm}$ off the ground. Two arms were enclosed by walls $30 \mathrm{~cm}$ high and the other two arms were exposed. As per previous methods, rats were placed at the juncture of the open and closed arms and the number of entries into, and the amount of time spent on, the open and closed arms was recorded during a 5-minute test. Time spent on the open arms was used as an index of anxiety and the total number of arm entries was used as a measure of motor activity since it has been found to positively correlate with other measures of motor activity including rearing behavior (Cruz et al. 1994, Rodgers \& Johnson 1995).

\section{Social interaction}

The social interaction task was used to assess exploratory and anxiety behavior associated with interacting with a novel conspecific (File 1980, Frye et al. 2000). Each member of a pair of rats (one experimental, one stimulus) was placed in the opposite corners of an open field $(76 \times 57 \times 35 \mathrm{~cm})$. The total duration of time that experimental rats engaged an ovariectomized stimulus rat in crawling over and under partner, sniffing of partner, following with contact, genital investigation of partner, tumbling, boxing, and grooming was recorded during a 5-minute test (Frye et al. 2000). An ovariectomized rat was utilized as the stimulus 
animal in order to avoid the exposure of experimental rats to vaginocervical stimulation, which might occur if a male had been used as the stimulus animal. The duration of time spent interacting with a conspecific is an index of anxiety behavior.

\section{Paced mating}

Paced mating was utilized over standard mating because of its greater ethological relevance and procedures were carried out as reported previously (McClintock \& Adler 1978, Erskine 1985, Frye \& Erskine 1990, Gans \& Erskine 2003). Paced mating tests were conducted in a chamber $(37.5 \times 75 \times 30 \mathrm{~cm})$, which was equally divided by a partition that had a small $(5 \mathrm{~cm}$ in diameter) hole in the bottom center, to allow a female free access to both sides of the chamber, but which prevented the larger stimulus male from moving between sides. Females were placed in the side of the chamber opposite the stimulus male. Rats were behaviorally tested for an entire ejaculatory series. Behaviors recorded were the frequency of mounts and intromissions that preceded an ejaculation. As well, the frequency (lordosis quotient = incidence of lordosis/number of mounts) and intensity (lordosis rating) of lordosis, quantified by rating of dorsiflexion on a scale of 0-3 (Hardy \& DeBold 1972) was recorded. The percentage of proceptive behaviors (i.e. hopping, darting, ear wiggling) that occurred prior to sexual contacts was recorded for report as a proceptivity quotient. The percentage of aggressive behaviors (i.e. vocalizations, kicks, attacks) females displayed prior to sexual contacts was also recorded and reported as an aggression quotient. Pacing measures included the percentage of times the female left the compartment containing the male after receiving a particular copulatory stimuli (\% exits after mounts, intromissions, and ejaculations) and latencies in seconds to return to the male compartment after these stimuli. The normal pattern of pacing behaviors for the percentage of exits and return latencies to be longer after more intensive stimulation (ejaculations $>$ intromissions $>$ mounts) was observed in the present study.

\section{Tissue collection}

Immediately following testing, whole brains and trunk blood were collected for later measurement of corticosterone, $\mathrm{E}_{2}, \mathrm{P}_{4}, \mathrm{DHP}$, and $3 \alpha, 5 \alpha$-THP. Trunk blood was centrifuged at $3000 \mathrm{~g}$ for $10 \mathrm{~min}$, and serum was stored in $1.5 \mathrm{ml}$ aliquot tubes at $-80^{\circ} \mathrm{C}$. The brains were rapidly frozen on dry ice and stored at $-80^{\circ} \mathrm{C}$ prior to $\mathrm{RIA}$.

\section{Tissue preparation}

Serum was thawed on ice and steroids extracted as described below. Because all brains were used for RIA, histological analyses were not possible. The brains were thawed and, during dissection, were visually examined for cannulae placement, which was commensurate with past placement confirmed by histology. In the past, we have administered $3 \alpha, 5 \alpha$-THP to VTA or nearby sites, including substantia nigra or central gray (Frye \& Rhodes 2006a, 2006b, 2008), and found that only administration to the VTA modulates exploratory, anxiety, and socio-sexual behavior. Following this, midbrain, hippocampus, striatum, cortex, and a control region (interbrain) were dissected and steroids were extracted as described below.

\section{RIA for steroid hormones}

$\mathrm{E}_{2}, \mathrm{P}_{4}, \mathrm{DHP}, 3 \alpha, 5 \alpha-\mathrm{THP}$, and corticosterone concentrations were measured as described below, using previously reported methods (Frye et al. 1996, Choi \& Dallman 1999, Frye \& Bayon 1999).

\section{Radioactive probes}

${ }^{3} \mathrm{H}_{2} \quad(\mathrm{NET}-317,51.3 \mathrm{Ci} / \mathrm{mmol}), \mathrm{P}_{4}$ (NET-208, specific activity $=47.5 \mathrm{Ci} / \mathrm{mmol}$ ), $3 \alpha, 5 \alpha$-THP (used for DHP and $3 \alpha, 5 \alpha$-THP; NET-1047, specific activity $=65.0 \mathrm{Ci} / \mathrm{mmol}$ ), and corticosterone (NET 182, specific activity $=48.2 \mathrm{Ci} / \mathrm{mmol}$ ) were purchased from Perkin-Elmer (Boston, MA, USA).

\section{Extraction of steroids from serum}

$E_{2}, P_{4}$, DHP, and $3 \alpha, 5 \alpha$-THP were extracted from serum with ether following incubation with water and 800 c.p.m. of ${ }^{3} \mathrm{H}$ steroid (Frye \& Bayon 1999). Corticosterone was extracted from serum by heating at $60{ }^{\circ} \mathrm{C}$ for $30 \mathrm{~min}$ (Choi \& Dallman 1999). After snap-freezing twice, test tubes containing steroid and ether were evaporated to dryness in a Savant vacuum speed drier. Dried-down tubes were reconstituted with phosphate assay buffer to the original serum volume. Between 90 and $95 \%$ of steroid was recovered from plasma.

\section{Extraction of steroids from brain tissues}

$\mathrm{E}_{2}, \mathrm{P}_{4}, \mathrm{DHP}$, and $3 \alpha, 5 \alpha$-THP were extracted from the brain tissues following homogenization with a glass/glass homogenizer in $50 \% \mathrm{MeOH}$ and $1 \%$ acetic acid. Tissues were centrifuged at $3000 \mathrm{~g}$ and the supernatant was chromatographed on Sepak cartridges equilibrated with $50 \% \mathrm{MeOH}: 1 \%$ acetic acid. Steroids were eluted with increasing concentrations of $\mathrm{MeOH}(50 \%$ $\mathrm{MeOH}$ followed by $100 \% \mathrm{MeOH})$. Solvents were removed using a speed drier. Samples were reconstituted in $300 \mu$ lassay buffer. Between 86 and $94 \%$ of steroid was recovered from brain tissue.

\section{Set-up and incubation of RIAs}

The range of the standard curves was $0-1000$ pg for $E_{2}, 0-8000$ pg for $\mathrm{P}_{4}, \mathrm{DHP}$, and $3 \alpha, 5 \alpha-\mathrm{THP}$, and 0-4 ng for corticosterone. Standards were added to assay buffer followed by the addition of the appropriate antibody (described below) and ${ }^{3} \mathrm{H}$ steroid. Total assay volumes were $800 \mu \mathrm{l}$ for $E_{2}$ and $P_{4}, 950 \mu l$ for DHP, $1250 \mu \mathrm{l}$ for $3 \alpha, 5 \alpha$-THP, and $900 \mu \mathrm{l}$ for corticosterone. All assays were incubated overnight at $4{ }^{\circ} \mathrm{C}$, except for $\mathrm{E}_{2}$ and corticosterone, which incubated at room temperature for 50 and 60 min respectively.

\section{Antibodies}

The $E_{2}$ antibody (E\#244, Dr G D Niswender, Colorado State University, Fort Collins, CO, USA) was used in a 1:40 000 dilution, which generally binds between $40 \%$ and $60 \%$ of $\left[{ }^{3} \mathrm{H}\right] \mathrm{E}_{2}$ (Frye \& Bayon 1999), and bound $48 \%$ in the present study. This $E_{2}$ antibody has negligible $(<1 \%)$ cross-reactivity with other steroid hormones, including esterone, $17 \alpha$-estradiol, $P_{4}$, and 17-hydroxyprogesterone. The $\mathrm{P}_{4}$ antibody ( $\# 3337$ from Dr G D Niswender, Colorado State University), used in a 1:30 000 dilution, typically binds between $30 \%$ and $50 \%$ of $\left[{ }^{3} \mathrm{H}\right] \mathrm{P}_{4}$ (Frye \& Bayon 1999), and bound $43 \%$ in the present study. The $P_{4}$ 
antibody has very low levels ( $<4 \%$ ) of cross-reactivity with DHP and $3 \alpha, 5 \alpha-$ THP (Niswender 1973). The DHP (X-947) and $3 \alpha, 5 \alpha-$ THP antibodies (\#921412-5, purchased from Dr Robert Purdy, Veterans Medical Affairs, La Jolla, CA, USA) were used in a 1:5000 dilution, typically bind between 40 and $60 \%$ of $\left[{ }^{3} \mathrm{H}\right] 3 \alpha, 5 \alpha$-THP (Frye \& Bayon 1999), and bound $51 \%$ in the present study. The DHP antibody cross-reacts with $3 \alpha, 5 \alpha$-THP (100\%), 5 $\alpha$-pregnan-3,20-dione (50\%), 4-pregnen-3 $\alpha$-ol-20-one (50\%), and $\mathrm{P}_{4}$ (17\%; Purdy et al. 1990). The $3 \alpha, 5 \alpha$-THPantibody cross-reacts with $3 \alpha$-hydroxypregn-4en-20-one (84\%) and DHP (11\%), and its $\beta$-isomer ( $7 \%), \mathrm{P}_{4}(6 \%)$, and pregnenolone $(<2 \%$; Purdy et al. 1990, Finn \& Gee 1994). The corticosterone antibody (\#B3-163, Endocrine Sciences, Tarzana, CA, USA), which typically binds $40-60 \%$ of $\left[{ }^{3} \mathrm{H}\right]$ corticosterone, was used in a 1:20 000 dilution and bound $45 \%$ in the present study.

\section{Termination of binding}

Separation of bound and free steroid was accomplished by the rapid addition of dextran-coated charcoal. Following incubation with charcoal, samples were centrifuged at $3000 \mathrm{~g}$ and the supernatant was decanted into a glass scintillation vial with $5 \mathrm{ml}$ scintillation cocktail. Sample tube concentrations were calculated using the logit-log method of Rodbard \& Hutt (1974), interpolation of the standards, and correction for recovery with 'AssayZap' interpolation software published by Biosoft (1994). The inter- and intra-assay reliability coefficients were: $E_{2}, 0.09$ and $0.10 ; P_{4}, 0.12$ and 0.13; DHP, 0.12 and $0.14 ; 3 \alpha, 5 \alpha-$ THP, 0.13 and 0.15 ; and corticosterone, 0.04 and 0.07 .

\section{Statistical analyses}

Each subject was tested once through the behavioral battery described yielding a factorial, between-group experimental design. Two independent variable conditions were examined: (1) $3 \alpha, 5 \alpha$-THP inhibitor condition - which had four levels (vehicle, PK11195, indomethacin, or PK11195+indomethacin) and (2) FGIN 1-27 condition - which had two levels (vehicle or FGIN 1-27). Two-way analyses of variance (ANOVA) were utilized to examine the effects of $3 \alpha, 5 \alpha$-THP inhibition and/or enhancement of $3 \alpha, 5 \alpha$-THP formation on endocrine and behavioral endpoints. Since engaging in paced mating is also expected to enhance biosynthesis of $3 \alpha, 5 \alpha$-THP (Frye \& Rhodes 2006a, Frye et al. 2007), simple regression analyses were also utilized to determine the extent to which brain concentrations of $3 \alpha, 5 \alpha$-THP may account for the aspects of behavioral responses (which occurred prior to sexual contact). $\alpha$-level for statistical significance was $P<0.05$. ANOVAs were followed by Fisher's protected least significant difference post hoc tests to ascertain group differences.

\section{Declaration of interest}

The authors declare that there is no conflict of interest that could be perceived as prejudicing the impartiality of the research reported.

\section{Funding}

This research was funded by a grant from the National Institute of Mental Health (MH06769801).

\section{Acknowledgements}

We thank Sanghamitra Dhalimbkar who assisted with data collection.

\section{References}

Avitsur R, Donchin O, Barak O, Cohen E \& Yirmiya R 1995 Behavioral effects of interleukin-1 $\beta$ : modulation by gender, estrus cycle, and progesterone. Brain, Behavior, and Immunity 9 234-241.

Birke LI \& Archer J 1975 Open-field behaviour of oestrous and dioestrous rats: evidence against an 'emotionality' interpretation. Animal Behaviour 23 509-512.

Bitran D, Foley M, Audette D, Leslie N \& Frye CA 2000 Activation of peripheral mitochondrial benzodiazepine receptors in the hippocampus stimulates allopregnanolone synthesis and produces anxiolytic-like effects in the rat. Psychopharmacology 151 64-71.

Blizard DA, Lippman HR \& Chen JJ 1975 Sex differences in open-field behavior in the rat: the inductive and activational role of gonadal hormones. Physiology and Behavior 14 601-608.

Brown RC \& Papadopoulos V 2001 Role of the peripheral-type benzodiazepine receptor in adrenal and brain steroidogenesis. International Review of Neurobiology 46 117-143.

Choi S \& Dallman MF 1999 Hypothalamic obesity: multiple routes mediated by loss of function in medial cell groups. Endocrinology $\mathbf{1 4 0}$ 4081-4088.

Cruz APM, Frei F \& Graeff FG 1994 Ethopharmacological analysis of rat behavior on the elevated plus-maze. Pharmacology, Biochemistry, and Behavior 49 171-176.

Dohi A, Dillon GH \& Singh M 2008 The role of progesterone and its metabolites in premenstrual disorders of affect. In Neuroactive Steroids in Brain Function, Behavior and Neuropsychiatric Disorders: Novel Strategies for Research and Treatment, pp 483-491. Eds MS Ritsner \& AW Weizman. Berlin: Springer.

Drugan RC, Basile AS, Ha JH \& Ferland RJ 1994 The protective effects of stress control may be mediated by increased brain levels of benzodiazepine receptor agonists. Brain Research 661 127-136.

Engel SR \& Grant KA 2001 Neurosteroids and behavior. International Review of Neurobiology 46 321-348.

Engin E \& Treit D 2007 The anxiolytic-like effects of allopregnanolone vary as a function of intracerebral microinfusion site: the amygdala, medial prefrontal cortex, or hippocampus. Behavioural Pharmacology 18 461-470.

Erskine MS 1985 Effects of paced coital stimulation on estrus duration in intact cycling rats and ovariectomized and ovariectomized-adrenalectomized hormone-primed rats. Behavioral Neuroscience 99 151-161.

Erskine MS \& Kornberg E 1992 Stress and ACTH increase circulating concentrations of $3 \alpha$-androstanediol in female rats. Life Sciences $\mathbf{5 1}$ 2065-2071.

Feder HH 1984 Hormones and sexual behavior. Annual Review of Psychology 35 165-200.

File SE 1980 The use of social interaction as a method for detecting anxiolytic activity of chlordiazepoxide-like drugs. Journal of Neuroscience Methods 2 219-238.

File SE 1990 New strategies in the search for anxiolytics. Drug Design and Delivery 5 195-201.

Finn DA \& Gee KW 1994 The estrus cycle, sensitivity to convulsants and the anticonvulsant effect of a neuroactive steroid. Journal of Pharmacology and Experimental Therapeutics 271 164-170.

Finn DA, Roberts AJ, Long S, Tanchuck M \& Phillips TJ 2003 Neurosteroid consumption has anxiolytic effects in mice. Pharmacology, Biochemistry, and Behavior 76 451-462.

Frye CA 2001a The role of neurosteroids and non-genomic effects of progestins and androgens in mediating sexual receptivity of rodents. Brain Research. Brain Research Reviews 37 201-222. 
Frye CA $2001 b$ The role of neurosteroids and nongenomic effects of progestins in the ventral tegmental area in mediating sexual receptivity of rodents. Hormones and Behavior 40 226-233.

Frye CA \& Bayon LE 1999 Mating stimuli influence endogenous variations in the neurosteroids $3 \alpha, 5 \alpha$-THP and $3 \alpha$-Diol. Journal of Neuroendocrinology 11 839-847.

Frye CA \& Erskine MS 1990 Influence of time of mating and paced copulation on induction of pseudopregnancy in cyclic female rats. Journal of Reproduction and Fertility 90 375-385.

Frye CA \& Petralia SM 2003a Mitochondrial benzodiazepine receptors in the ventral tegmental area modulate sexual behaviour of cycling or hormone-primed hamsters. Journal of Neuroendocrinology 15 677-686.

Frye CA \& Petralia SM 2003b Lordosis of rats is modified by neurosteroidogenic effects of membrane benzodiazepine receptors in the ventral tegmental area. Neuroendocrinology 77 71-82.

Frye CA \& Rhodes ME 2006a Infusions of $5 \alpha$-pregnan-3 $\alpha$-ol-20-one $(3 \alpha, 5 \alpha$ THP) to the ventral tegmental area, but not the substantia nigra, enhance exploratory, anti-anxiety, social and sexual behaviours and concomitantly increase $3 \alpha, 5 \alpha$-THP concentrations in the hippocampus, diencephalon and cortex of ovariectomised oestrogen-primed rats. Journal of Neuroendocrinology 18 960-975.

Frye CA \& Rhodes ME 2006b Progestin concentrations are increased following paced mating in midbrain, hippocampus, diencephalon, and cortex of rats in behavioral estrus, but only in midbrain of diestrous rats. Neuroendocrinology 83 336-347.

Frye CA \& Rhodes ME 2008 Infusions of $3 \alpha, 5 \alpha$-THP to the VTA enhance exploratory, anti-anxiety, social, and sexual behavior and increase levels of $3 \alpha, 5 \alpha$-THP in midbrain, hippocampus, diencephalon, and cortex of female rats. Behavioural Brain Research 187 88-99.

Frye CA \& Vongher JM 2001 Ventral tegmental area infusions of inhibitors of the biosynthesis and metabolism of $3 \alpha, 5 \alpha$-THP attenuate lordosis of hormone-primed and behavioural oestrous rats and hamsters. Journal of Neuroendocrinology 13 1076-1086.

Frye CA \& Walf AA 2008 Membrane actions of progestins at dopamine type 1-like and $\operatorname{GABA}(\mathrm{A})$ receptors involve downstream signal transduction pathways. Steroids 73 906-913.

Frye CA, McCormick CM, Coopersmith C \& Erskine MS 1996 Effects of paced and non-paced mating stimulation on plasma progesterone, $3 \alpha$ diol and corticosterone. Psychoneuroendocrinology 21 431-439.

Frye CA, Petralia SM \& Rhodes ME 2000 Estrous cycle and sex differences in performance on anxiety tasks coincide with increases in hippocampal progesterone and $3 \alpha, 5 \alpha$-THP. Pharmacology, Biochemistry, and Behavior 67 587-596.

Frye CA, Rhodes ME, Petralia SM, Walf AA, Sumida K \& Edinger KL $20063 \alpha$ Hydroxy-5 $\alpha$-pregnan-20-one in the midbrain ventral tegmental area mediates social, sexual, and affective behaviors. Neuroscience 138 1007-1014.

Frye CA, Paris JJ \& Rhodes ME 2007 Engaging in paced mating, but neither exploratory, anti-anxiety, nor social behavior, increases $5 \alpha$-reduced progestin concentrations in midbrain, hippocampus, striatum, and cortex. Reproduction 133 663-674.

Gans S \& Erskine MS 2003 Effects of neonatal testosterone treatment on pacing behaviors and development of a conditioned place preference. Hormones and Behavior 44 354-364.

Hall PF 1985 Steroidogenic cytochromes P450: do the properties of the homogeneous enzymes reveal important aspects of the regulation of steroid synthesis in vivo? Endocrine Research 10 311-317.

Hardy DF \& DeBold JF 1972 Effects of coital stimulation upon behavior of the female rat. Journal of Comparative and Physiological Psychology $\mathbf{7 8}$ $400-408$.

Liang S, Byersa DM \& Irwina LN 2008 Sex and diet affect the behavioral response of rats to chronic mild stressors. Physiology and Behavior 28 27-36.

Long JA \& Evans HM 1922 Oestrous cycle in the rat and its associated phenomena. Memoirs of the University of California 6 1-146.
Marshall JF \& Teitelbaum P 1974 Further analysis of sensory inattention following lateral hypothalamic damage in rats. Journal of Comparative and Physiological Psychology 86 375-395.

McClintock MK \& Adler NT 1978 Induction of persistent estrus by airborne chemical communication among female rats. Hormones and Behavior $11414-418$

Mellon SH, Griffin LD \& Compagnone NA 2001 Biosynthesis and action of neurosteroids. Brain Research. Brain Research Reviews 37 3-12.

Micevych P, Soma KK \& Sinchak K 2007 Neuroprogesterone: key to estrogen positive feedback? Brain Research Reviews 57 470-480.

Otto S, Bhattacharyya KK \& Jefcoate CR 1992 Polycyclic aromatic hydrocarbon metabolism in rat adrenal, ovary, and testis microsomes is catalyzed by the same novel cytochrome P450 (P450RAP). Endocrinology 131 3067-3076.

Patchev VK, Shoaib M, Holsboer F \& Almeida OF 1994 The neurosteroid tetrahydroprogesterone counteracts corticotropin-releasing hormoneinduced anxiety and alters the release and gene expression of corticotropinreleasing hormone in the rat hypothalamus. Neuroscience 62 265-271.

Patchev VK, Hassan AH, Holsboer DF \& Almeida OF 1996 The neurosteroid tetrahydroprogesterone attenuates the endocrine response to stress and exerts glucocorticoid-like effects on vasopressin gene transcription in the rat hypothalamus. Neuropsychopharmacology $\mathbf{1 5}$ 533-540.

Plassart-Schiess E \& Baulieu EE 2001 Neurosteroids: recent findings. Brain Research. Brain Research Reviews 37 133-140.

Purdy RH, Moore PH Jr, Rao PN, Hagino N, Yamaguchi T, Schmidt P, Rubinow DR, Morrow AL \& Paul SM 1990 Radioimmunoassay of $3 \alpha-$ hydroxy-5 $\alpha$-pregnan-20-one in rat and human plasma. Steroids $\mathbf{5 5}$ 290-296.

Purdy RH, Morrow AL, Moore PH Jr \& Paul SM 1991 Stress-induced elevations of gamma-aminobutyric acid type A receptor-active steroids in the rat brain. PNAS $\mathbf{8 8} 4553-4557$.

Renard GM, Rivarolaa MA \& Suáreza MM 2007 Sexual dimorphism in rats: effects of early maternal separation and variable chronic stress on pituitary-adrenal axis and behavior. International Journal of Developmental Neuroscience 25 373-379.

Rodbard D \& Hutt DM 1974 Statistical analysis of radioimmunoassay and immunoradiometric assays: a generalized, weighted iterative, least squares method for logistic curve fitting. In Symposium on Radioimmunoassay and Related Procedures in Medicine. Uniput, NY, pp 209-233. Ed. International Atomic Energy Agency. Vienna: International Atomic Energy Agency.

Rodgers RJ \& Johnson JT 1995 Factor analysis of spatiotemporal and ethological measures in the murine elevated plus-maze test of anxiety. Pharmacology, Biochemistry, and Behavior 52 297-303.

Walf AA \& Frye CA 2003 Anti-nociception following exposure to trimethylthiazoline, peripheral or intra-amygdala estrogen and/or progesterone. Behavioural Brain Research 144 77-85.

Walf AA \& Frye CA 2007 Estradiol decreases anxiety behavior and enhances inhibitory avoidance and gestational stress produces opposite effects. Stress 10 251-260.

Walf AA, Sumida K \& Frye CA 2006 Inhibiting $5 \alpha$-reductase in the amygdala attenuates antianxiety and antidepressive behavior of naturally receptive and hormone-primed ovariectomized rats. Psychopharmacology 186 $302-311$

Received 5 June 2008

First decision 25 June 2008

Revised manuscript received 4 September 2008

Accepted 25 September 2008 